\title{
Cancer prevention by aspirin in children with Constitutional Mismatch Repair Deficiency (CMMRD)
}

\author{
Erika K. S. M. Leenders ${ }^{1} \cdot$ Harm Westdorp ${ }^{2,3} \cdot$ Roger J. Brüggemann $^{4} \cdot$ Jan Loeffen $^{5,6} \cdot$ Christian Kratz $^{7} \cdot$ John Burn $^{8}$. \\ Nicoline Hoogerbrugge ${ }^{1} \cdot$ Marjolijn C. J. Jongmans ${ }^{1,6,9}$
}

Received: 8 November 2017 / Revised: 18 May 2018 / Accepted: 22 May 2018 / Published online: 14 June 2018

(c) European Society of Human Genetics 2018

\begin{abstract}
Constitutional MisMatch Repair Deficiency (CMMRD) is caused by homozygous or compound heterozygous germline variants in one of the mismatch repair (MMR) genes (MSH2, MSH6, PMS2, MLH1). This syndrome results in early onset colorectal cancer, leukemia and lymphoma, brain tumors and other malignancies. Children with CMMRD are at high risk of developing multiple cancers and cancer surveillance does not guarantee detection of cancer at a curable stage. The development of a preventive treatment strategy would be a major step forward. Long-term daily use of acetylsalicylic acid (ASA) has been shown to reduce cancer risk in individuals with Lynch syndrome (LS). LS is caused by heterozygous germline variants of $M S H 2, M S H 6, P M S 2$ and $M L H 1$ and characterized by an increased risk of developing colorectal and endometrial cancer at adult age. Here we discuss the potential use of ASA for cancer prevention in patients with CMMRD.
\end{abstract}

\section{Clinical case}

An eight-year-old boy was diagnosed with T-cell lymphoblastic lymphoma and treated according to I-BFM Euro-LB02 protocol. Because the patient had numerous cafe-au-laitlike spots, a clinical geneticist was consulted, who considered the diagnosis of constitutional mismatch repair deficiency syndrome (CMMRD) and Neurofibromatosis type 1. The diagnosis CMMRD was confirmed by the identification of compound heterozygous variants (c.[137 G > T]; [247_250dup], p.[Ser46Ile];[Thr84fs]) in the PMS2 gene (NM_000535.5) in blood derived DNA. An intensive surveillance program was started [1].

Marjolijn C. J. Jongmans

marjolijn.jongmans@ radboudumc.nl

1 Department of Human Genetics, Radboud University Medical Center, Radboud Institute for Molecular Life Sciences, Nijmegen, The Netherlands

2 Department of Tumor Immunology, Radboud Institute for Molecular Life Sciences, Radboud University Medical Center, Nijmegen, The Netherlands

3 Department of Medical Oncology, Radboud University Medical Center, Nijmegen, The Netherlands

4 Department of Pharmacy, Radboud University Nijmegen Medical Centre, Radboud Institute for Health Sciences, Nijmegen, The
The parents of the child suggested the use of acetylsalicylic acid (ASA, aspirin) as a preventive drug for colorectal cancer, based on studies in adult patients with Lynch syndrome (LS), which is caused by heterozygous mismatch repair (MMR) gene variants. In these studies reductions in the incidence of colorectal cancer (CRC) are described among chronic users of ASA with LS [2]. After careful consideration with a multidisciplinary team continuous treatment with ASA at a dosage of $100 \mathrm{mg}$ a day was started at the beginning of the maintenance phase of treatment for T-cell lymphoblastic lymphoma (consisting of once weekly oral methotrexate (MTX) and once daily oral 6-mercaptopurine). On the day of MTX intake, no ASA was given because of the potential interaction; i.e., ASA can

Netherlands

5 Department of Pediatric Oncology, Erasmus MC - Sophia Children's Hospital, Rotterdam, The Netherlands

6 Princess Máxima Center for Pediatric Oncology, Utrecht, The Netherlands

7 Pediatric Hematology and Oncology, Hannover Medical School, Hannover, Germany

8 Institute of Genetic Medicine Newcastle University, Newcastle upon Tyne, UK

9 Department of Medical Genetics, University Medical Center Utrecht, Utrecht, The Netherlands 
lower the clearance of MTX and thus exacerbate its toxicity [3]. Liver and kidney function was closely monitored. The patient suffered from isolated testicular relapse of T-NHL one year after start of maintenance therapy. There were no other localizations of T-NHL at time of relapse. He received reinduction chemotherapy followed by allogeneic bone marrow transplantation of an HLA identical sibling. Currently he is alive and well and 3.7 years in second complete remission. He is currently 5.5 years after start of ASA without adverse events. Annual gastro duodenoscopy and coloscopy showed no signs of (premalignant) gastrointestinal (GI) cancer.

The rationale for the use of ASA in patients with CMMRD is that the drug may potentially reduce the cancer risk outweighing possible ASA side effects. Due to small numbers, a prospective study on the effects of ASA in patients with CMMRD is unlikely. Here, we discuss the potential of ASA for cancer prevention in CMMRD and provide all available information for considering implementation in clinical practice.

\section{CMMRD}

CMMRD, a recessive pediatric cancer predisposition syndrome, has been first described in 1999 [4, 5]. Mainly three types of malignancy characterize this syndrome: CRC, leukemia and lymphoma and brain tumors. These cancers occur in childhood or adolescence. If patients survive the first cancer, they have a high chance of developing a second or even a third malignancy. A variety of non-malignant lesions have also been observed in CMMRD, such as hyperand hypo-pigmented lesions of the skin, mild immunoglobulin deficiencies and rarely congenital malformations [1].

CMMRD is caused by homozygous or compound heterozygous germline variants in one of the DNA MMR genes (i.e., $M L H 1, M S H 2$, MSH6, PMS2). A list of known disease-causing variants in these genes can be found in the Leiden Open Variation Database (www.lod.nl). Congenital deficiency of the MMR system in all cells of the body explains the high cancer risk in these children and results in ultra-hypermutated cancers [6]. In most cases both parents of children with CMMRD carry a heterozygous germline variant, which leads to the autosomal dominant condition LS. The main clinical features of LS are adult onset CRC and endometrial cancer, with an average age at diagnosis of 44-61 years for CRC and 48-62 years for endometrial cancer in women [7]. In addition, LS patients are at an increased risk of developing malignancies in the ovaries, stomach, small intestines, urinary tract and sebaceous glands [8]. Tumors that develop as a result of LS can effectively be recognized by microsatellite instability (MSI). MSI is a somatic accumulation of length variations in repetitive DNA sequences resulting from MMR deficiency and is strongly correlated with the development of cancer in LS patients [9].

Because of the large cancer risk in CMMRD, children with this condition should follow an intensive surveillance program. Durno et al. published the outcome of surveillance in two sisters with CMMRD [10]. Fifteen cancers were detected over a follow-up period of 10 years, including a jejunal carcinoma and a small asymptomatic anaplastic astrocytoma that could be completely resected. Recently, expert opinion based surveillance guidelines were proposed by the European Consortium 'Care for CMMRD' and by the 'US Multi-Society Task Force on Colorectal Cancer' [1, 11]. Structural data collection at an international scale is needed to collect evidence on the effects of this guideline on morbidity and survival. Bakry and colleagues have performed such a study. They followed 23 children for a median period of 61 months and detected 39 malignant and premalignant lesions in total, including asymptomatic malignant gliomas and GI carcinomas, suggesting that surveillance in CMMRD may add to early detection [12].

Surveillance however does not guarantee the detection of precancerous lesions or cancer at a curable stage. A preventive treatment strategy with ASA would therefore be a major step forward.

\section{ASA for cancer prevention}

ASA is known as an analgesic and anti-inflammatory drug. The antiplatelet effects of ASA made the drug also suitable for reducing vascular morbidity and mortality. Observational studies, across a range of cancer sites, revealed an association between ASA use and reduced cancer incidence, particularly for CRC and gastro-esophageal cancer [13, 14]. The first two randomized controlled trials on the effect of ASA on risk of cancer as primary endpoint revealed apparently negative findings $[15,16]$. The first, the Women's Health Study (WHS) used every low dose aspirin in unselected women and showed no effect at 10 years. The second, discussed below, focused on Lynch syndrome carriers and included 10 year blinded follow up in view of early observational reports of delayed effect. At first follow up, a greater than 50\% reduction in colorectal and other cancers was apparent.

Retracting of people who took part in the early trials of aspirin as a cardiovascular protective drug revealed a major reduction in CRC and other cancer mortality after 10 years of follow-up [17, 18]. This prompted extended follow up of the WHS, which revealed an $18 \%$ reduction in CRC beyond the end of the trial [19]. ASA also has a positive influence on the short-term risk of dying from cancer $[20,21]$. These and other findings suggest that ASA can potentially reduce cancer growth and/or affect the development and spread of 
metastases. A thorough review of the association between ASA, cancer prevention and cancer outcome is provided by Langley and Rothwell [22].

\section{ASA for cancer prevention in Lynch syndrome}

The CAPP2 study was designed to test the hypothesis that the use of ASA in individuals with LS reduces the development of CRC (as primary outcome) and other LS cancers (as secondary outcome). Adult LS patients were randomized to $600 \mathrm{mg}$ of ASA $(n=427)$ or placebo $(n=434)$, for up to 4 years. Overall, 48 participants were diagnosed with CRC in the follow-up period. Of these, 18 received ASA and 30 received placebo. These results showed a nonsignificant effect favouring ASA $(P=0.12)$. However, subgroup analysis suggests a delayed effect of ASA. For participants completing $\geq 2$ years of intervention, there was a reduction in risk of $\mathrm{CRC}$ (hazard ratio $=0.41, \mathrm{CI}$ : $0.19-0.86, P=0.02$ ) and a similar impact on overall numbers of LS cancers. The key conclusion from this study was, therefore, that taking $600 \mathrm{mg}$ ASA daily for at least two years results in a reduction of $63 \%$ in CRC in people with LS, but this effect does not become apparent until about 5 years from the beginning of the intervention. Lower doses of ASA (100 and $300 \mathrm{mg}$ daily) are now being evaluated in the CAPP 3 trial, with a non-inferiority design and ASA $600 \mathrm{mg}$ daily as the standard of care [23, 24].

\section{Potential mechanisms of action}

ASA belongs to the group of drugs called non-steroidal anti-inflammatory drugs (NSAIDs) and acts via the inhibition of cyclooxygenase (COX) enzymes resulting in decreased prostaglandin synthesis [25, 26]. One isoform of COX, named COX-2, promotes cell proliferation and inflammation [25, 27]. In CRC, overexpression of the COX2 enzyme is frequently observed [25, 28, 29]. CRC prevention by ASA is mediated through various mechanisms, including the inhibition of COX-2 and prostaglandin synthesis, interfering in the host's immune response and through inactivation of platelets [30].

Prostaglandins can influence different immune pathways and immunosuppression is observed in tissues where there is a high prostaglandin E2 production, resulting in negatively regulated $\mathrm{T}$-cell proliferation, cytokine production, and cytotoxicity potential [31]. It is therefore postulated that ASA may enhance antitumor T-cell activity and influence the degree of tumor-infiltrating lymphocytes. In a recent study, using two prospective cohorts, regular ASA use indeed lowered the risk of CRC with low numbers of tumor- infiltrating lymphocytes, which seemed to be independent of MSI status [32]. The mechanism might be that ASA, either related or unrelated to the inhibition of prostaglandins, inhibits cancer development by restoring T-cellmediated antitumor immunity [33].

The antiplatelet effect of ASA can be a potential mechanism of action in cancer prevention. The antithrombotic effect of aspirin is not dose related, an observation consistent with saturability of platelet COX-1 inhibition by aspirin at very low doses. Also for the cancer and adenoma chemopreventive effect of aspirin saturability is observed at low doses (75-100 mg taken daily or every other day) [19, $34,35]$, suggesting that acetylation of platelet COX-1 is an important mechanism of action of aspirin in preventing cancer. There is increasing knowledge about the interaction between platelets and T-cell immunity against cancer. Platelets promote malignancy and resistance to therapy by suppressing T-cell activation and function [36-38]. A recent study shows that platelets downregulate T-cell immunity, specifically CD4 + and CD $8+\mathrm{T}$ cells, via transforming growth factor $\beta$ (TGF $\beta$ ) and to a lesser extent through lactate [38]. Platelets are an important source of functional TGF $\beta$, systemically as well as in the tumor microenvironment, primarily through the expression of the cell surface TGF $\beta$-docking receptor Glycoprotein A Repetitions Predominant (GARP). Platelet-related TGF $\beta$ activation contributes dominantly to the immunosuppressive effect in cancer. This is supported by enhanced tumorspecific T-cell immunity in mice with platelet-specific deletion of GARP [38].

The mechanisms described above are hypothetical for CRC in general. However, specific mechanisms of action affecting microsatellite unstable cells is important for the cancer preventive function of ASA in LS. The DNA MMR pathway recognizes and repairs nucleotide mispairs. Deficiency of this system results in the accumulation of multiple secondary variants that drive tumorigenesis. In tumor tissue of LS patients this is displayed by MSI. In MMR-deficient human CRC cell lines, it was shown that ASA appears to enhance apoptosis in critically unstable cells and thereby suppresses the MSI mutator phenotype [39, 40]. In other words: ASA might delete the aberrant stem cells that are most likely to progress rapidly to cancer. In vitro ASA has been shown to arrest CRC cells at the G1/S checkpoint and to induce apoptosis through activation of ATM, p21, and BAX [41].

\section{Adverse effects of ASA use in children}

For obvious reasons adverse events of the drug, in particular if used daily and long term, should be taken into account. Indications for chronic ASA use in children are autoimmune conditions like Kawasaki disease and rheumatologic 
disorders and for inhibition of platelet adhesiveness to prevent thrombotic events.

A frequent side effect is an increased risk of bleeding events. This is an important concern in young children who are prone to falling and older children who participate in contact sports. Very little data on the safety of antiplatelet therapy for pediatric patients are available, and most of what is practiced is based on adult studies. In one study ASA therapy (a daily dose of $2-5 \mathrm{mg} / \mathrm{kg}$ for 3-6-months) was monitored in children after international cardiac catheterization. No major bleeding event (i.e., GI or intracranial bleeding or bleeding requiring transfusion) was observed. Still, $19 \%$ of children had minor bleeding symptoms, and of those, the median age was significantly lower than that of children without bleeding [42]. In the CAPP1 study children and adolescents from 10 to 21 years of age with familial adenomatous polyposis (FAP) were treated with $600 \mathrm{mg}$ of ASA (max. $300 \mathrm{mg}$ for those under 12 years). In this study, which did not reveal a significant effect on polyp formation, no serious adverse effects were recorded [2].

ASA use is also associated with upper GI side effects, including peptic ulcers. Co-administration of a proton pump inhibitor (PPI) is an effective therapy for the prevention or relief of these ASA-induced side effects [43]. However, chronic use of a PPI also has its risks, for example an enhanced risk of chronic kidney disease and higher risk of infectious complications due to removal of an acidic environment and therefore natural barrier as defense against pathogens $[44,45]$. In the CMMRD case described above, it was decided to prescribe a PPI to reduce the risk of potential ASA side effects.

A very rare side effect of ASA in children is Reye's hepatic encephalopathy [46], an acute illness characterized by encephalopathy and fatty degeneration of the liver. Reye's syndrome occurs almost exclusively in children with an estimated incidence of $0.8 / 1,000,000$ children [47]. The precise cause of Reye's syndrome is unknown, but the condition is often preceded by an acute viral infection. Rates of Reye's syndrome fell dramatically following the identification of ASA use as a risk factor and advisories against use of ASA in febrile children [48-50]. A retrospective study of 26 survivors of Reye's syndrome revealed that the majority of the patients $(n=18,69 \%)$ had various metabolic disorders [51-53].

Children with CMMRD might be more prone to serious adverse effects of ASA use because they are at risk for bleeding events in cancerous lesions. This is not necessarily detrimental for the patient, since fecal blood for instance can trigger awareness for an internal colon carcinoma, i.e., a carcinoma that has developed between two surveillance colonoscopies. A hemorrhage in a brain tumor however, can have serious complications.
Overall, we conclude that the potential benefits of ASA for children with CMMRD outweigh the almost negligible chances of developing Reye's syndrome. The bleeding risks and GI side effects, however, should be taken into account and may ask for behavioral changes or other preventive strategies such as prescribing a PPI or monitoring of relevant blood results. Side effects of ASA are in part dosedependent and, therefore, the lowest effective dose should be administered.

\section{Starting age}

In observational studies, the protection against cancer in regular ASA users took about ten years to emerge [14, 54]. This delayed effect is consistent with the hypothesis that ASA has an effect on the early stages of carcinogenesis. A still delayed, but considerably faster effect of ASA on the incidence of CRC was observed in the CAPP2 study with an effect becoming apparent 3-4 years from the start of ASA intervention [2]. The authors stated that this difference is consistent with faster colon cancer (CRC) development in individuals with LS compared to sporadic CRC. If this hypothesis can be extrapolated to CMMRD, an even faster effect of cancer prevention by ASA would be expected in children with CMMRD, based on the extreme pace at which cancers develop in these children [6].

Given the apparent delay in the chemopreventive effect of ASA and the young age at which cancers have been observed in children with CMMRD, the optimum moment to start treatment might be at birth or soon thereafter. This is in conflict with the current clinical diagnostic criteria postulated by the European consortium Care for CMMRD [1]. These criteria are developed to detect CMMRD among children who already did develop cancer and do not assist in recognizing these children before they are affected by cancer. If compelling evidence would be available for the preventive potential of ASA in children with CMMRD, this would justify to screen for CMMRD in all children with multiple ( $>5$ ) unexplained hyperpigmentations of the skin, hence also in children without a malignancy. At present, we advise considering administration of ASA as soon as CMMRD is diagnosed. Genetic counselling and testing is recommended for the extended family of a cancer patient with CMMRD in order to identify additional variant carriers who may benefit from this preventive strategy combined with cancer surveillance.

\section{Dose}

Evidence is lacking for a dose-related effect of aspirin in preventing cancer in patients with Lynch syndrome. In the 
CAPP2 study, $600 \mathrm{mg}$ ASA was compared to placebo in an adult study population [2]. Results of the CAPP3 study, which compares the efficacy of $300 \mathrm{mg}$ and $100 \mathrm{mg}$ have yet to be published, but also encompasses adult study participants [23]. Choosing an appropriate dose in children is challenging. ASA is well and almost fully absorbed after oral administration in the proximal part of the duodenum. After absorption it is transferred to salicylic acid in the liver and to a lesser extent in plasma and erythrocytes. CYP2C9 and UGT1A6 are the dominant metabolic pathways. Salicylic acid and the metabolites are excreted by urine. Drug clearance in pediatrics may be influenced by weight, age, and protein binding (90-95\% is protein bound). In addition renal function may impact clearance of metabolites, but how these factors interact is not easily determined.

As a consequence, the challenge lies in finding an equivalent dose for pediatric patients that results in similar exposure to adults assuming the pharmacodynamic effect in children will be identical to that in adults. In children, ASA is usually administered at a dose ranging from 2 to $10 \mathrm{mg} /$ $\mathrm{kg}$ /day; higher ASA doses are only used as antiinflammatory treatment for children with Kawasaki disease [55]. However, there are limitations to this model, such as a nonlinear relationship between weight and drug elimination. The linear per kilogram model, therefore, underestimates clearance and, consequently, maintenance dose in children. An alternative approach is the allometric model, in which an exponent of weight is used for scaling drug clearance. This model disentangles size from age, allowing a consistent approach to describing data in children and adults [56]. We consider this model more appropriate to determine the appropriate ASA dose in CMMRD children.

In the CAPP2 study in adults with LS, $600 \mathrm{mg} \mathrm{ASA}$ was compared to placebo [2]. The key conclusion from this study was that taking $600 \mathrm{mg}$ ASA daily could lead to a CRC reduction of $63 \%$. Even after allometric scaling, an adult dose of $600 \mathrm{mg}$ leads to very high doses of ASA in children; i.e., for a child of $10 \mathrm{~kg}$ a dose of $140 \mathrm{mg}$ should then be administered. This dosage is much higher than the usual ASA dosage in, for example, Kawasaki disease. Therefore, we advise using an allometric model for dose calculation based on an adult dose of $300 \mathrm{mg}$, assuming a body weight of $70 \mathrm{~kg}$. Dose estimation will be influenced by the outcome of the dose non-inferiority trial (CAPP3) [23].

\section{Interactions with other drugs}

Since CMMRD syndrome is often recognized in children who were recently diagnosed with cancer, preventive treatment with ASA may need to be considered while the patient receives cancer chemotherapy. Chronic use of ASA may therefore lead to important interactions with common drugs used in cancer treatment. However, with low doses of ASA, very few interactions have been reported.

Drug interactions can be categorized as either pharmacokinetic or pharmacodynamic. Pharmacokinetic interactions involve for instance drug-metabolizing enzymes or drug-transporters (42). Prostaglandin synthesis inhibitors, and therefore also high doses of ASA, can potentially lower the clearance of MTX and thus exacerbate its toxicity [3]. However, this has not been investigated in pediatric patients. In the CMMRD case described above, it was decided to skip ASA treatment on days of MTX treatment, as this will not likely lower the overall preventive effect of ASA.

Pharmacodynamic interactions result from concomitant administered drugs with similar toxicity profiles. This could lead to identical side effects or opposing effects in which case the overall effect is decreased or 'canceled out'. Pharmacodynamic interactions with ASA, for example, involve the combination with glucocorticoids, increasing the potential for peptic ulcera and bleeding events [57]. Other examples include the interaction with warfarin (coumarin derivatives) or Direct Acting Oral AntiCoagulants, that increase the risk of bleeding disorders, or the combination with ibuprofen (NSAID), that diminishes the effect of ASA [57, 58].

Drug-drug interactions from ASA with concomitant use of oncolytic drugs in pediatric patients are not (yet) described. Combination with other chemotherapy should be carefully introduced and consulting a clinical pharmacist might be considered.

\section{Recommendations}

Sufficient data to recommend the use of ASA as a preventive measure in patients with CMMRD is lacking. Here we have provided all currently available information that is needed to perform a risk-benefit analysis for application of ASA in an individual patient with CMMRD. If ASA is prescribed, families and physicians should be aware of the potentially increased risk of hemorrhage (e.g., bleeding of brain tumors). Data on the use of ASA and potential complications should be made available to CMMRD registries.

\section{Compliance with ethical standards}

Conflict of interest Professor Burn has a longstanding relationship with Bayer Pharma who have supported the CaPP Cancer Prevention trials. He has received fees for delivering lectures on this topic.

\section{References}

1. Vasen HF, Ghorbanoghli Z, Bourdeaut F, et al. Guidelines for surveillance of individuals with constitutional mismatch 
repair-deficiency proposed by the European Consortium "Care for CMMR-D” (C4CMMR-D). J Med Genet. 2014;51:283-93.

2. Burn J, Gerdes AM, Macrae F, Mecklin JP, Moeslein G, Olschwang S, et al. Long-term effect of aspirin on cancer risk in carriers of hereditary colorectal cancer: an analysis from the CAPP2 randomised controlled trial. Lancet. 2011;378:2081-7.

3. Bourre-Tessier J, Haraoui B. Methotrexate drug interactions in the treatment of rheumatoid arthritis: a systematic review. J Rheumatol. 2010;37:1416-21.

4. Ricciardone MD, Ozcelik T, Ceher B, et al. Human MLH1 deficiency predisposes to hematological malignancy and neurofibromatosis type 1. Cancer Res. 1999;59:290-3.

5. Wang Q, Lasset C, Desseigne F, Frappaz D, et al. Neurofibromatosis and early onset of cancers in hMLH1-deficient children. Cancer Res. 1999;59:294-7.

6. Shlien A, Campbell BB, de Borja R, et al. Combined hereditary and somatic mutations of replication error repair genes result in rapid onset of ultra-hypermutated cancers. Nat Genet. 2015; 47:257-62.

7. Kohlmann W, Gruber SB. Lynch Syndrome. In: Pagon RA, et al. Eds. GeneReiews(R). Seattle (WA): University of Washington; 1993.

8. Lynch HT, de la Chapelle A. Hereditary colorectal cancer. N Engl J Med. 2003;348:919-32.

9. Manders P, Spruijt L, Kets CM, et al. Young age and a positive family history of colorectal cancer are complementary selection criteria for the identification of Lynch syndrome. Eur J Cancer. 2011;47:1407-13.

10. Durno CA, Aronson M, Tabori U, Malkin D, Gallinger S, Chan HS. Oncologic surveillance for subjects with biallelic mismatch repair gene mutations: 10 year follow-up of a kindred. Pediatr Blood Cancer. 2012;59:652-6.

11. Durno C, Boland CR, Cohen S, et al. Recommendations on Surveillance and Management of Biallelic Mismatch Repair Deficiency (BMMRD) syndrome: a consensus statement by the US multi-society task force on colorectal cancer. Gastroenterology. 2017;152:1605-14.

12. Bakry D, Aronson M, Durno C, et al. Genetic and clinical determinants of constitutional mismatch repair deficiency syndrome: report from the constitutional mismatch repair deficiency consortium. Eur J Cancer. 2014;50:987-96.

13. Bosetti C, Rosato V, Gallus S, Cuzick J, La Vecchia C. Aspirin and cancer risk: a quantitative review to 2011. Ann Oncol. 2012;23:1403-15.

14. Cuzick J, Otto F, Baron JA, et al. Aspirin and non-steroidal antiinflammatory drugs for cancer preention: an international consensus statement. Lancet Oncol. 2009;10:501-7.

15. Cook NR, Lee IM, Gaziano JM, et al. Low-dose aspirin in the primary prevention of cancer: the Women's Health Study: a randomized controlled trial. JAMA. 2005;294:47-55.

16. Burn J, Bishop DT, Mecklin JP, Macrae F, et al. Effect of aspirin or resistant starch on colorectal neoplasia in the Lynch syndrome. N Engl J Med. 2008;359:2567-78.

17. Flossmann E, Rothwell PM. Effect of aspirin on long-term risk of colorectal cancer: consistent evidence from randomised and observational studies. Lancet. 2007;369:1603-13.

18. Rothwell PM, Wilson M, Elwin CE, et al. Long-term effect of aspirin on colorectal cancer incidence and mortality: 20-year follow-up of five randomised trials. Lancet. 2010;376:1741-50.

19. Cook NR, Lee IM, Zhang SM, Moorthy MV, Buring JE. Alternate-day, low-dose aspirin and cancer risk: long-term observational follow-up of a randomized trial. Ann Intern Med. 2013;159:77-85.

20. Rothwell PM, Price JF, Fowkes FG, et al. Short-term effects of daily aspirin on cancer incidence, mortality, and non-vascular death: analysis of the time course of risks and benefits in 51 randomised controlled trials. Lancet. 2012;379:1602-12.

21. Rothwell PM, Wilson M, Price JF, Belch JF, Meade TW, Mehta Z. Effect of daily aspirin on risk of cancer metastasis: a study of incident cancers during randomised controlled trials. Lancet. 2012;379:1591-601.

22. Langley RE, Rothwell PM. Aspirin in gastrointestinal oncology: new data on an old friend. Curr Opin Oncol. 2014;26:441-7.

23. Burn J, Mathers JC, Bishop DT. Chemopreention in Lynch syndrome. Fam Cancer. 2013;12:707-18.

24. Burn J, Sheth $\mathrm{H}$. The role of aspirin in preventing colorectal cancer. Br Med Bull. 2016;119:17-24.

25. Chan AT, Ogino S, Fuchs CS. Aspirin and the risk of colorectal cancer in relation to the expression of COX-2. N Engl J Med. 2007;356:2131-42.

26. Fink SP, Yamauchi M, Nishihara R, et al. Aspirin and the risk of colorectal cancer in relation to the expression of 15hydroxyprostaglandin dehydrogenase (HPGD). Sci Transl Med. 2014;6:233re2.

27. Temraz S, Mukherji D, Shamseddine A. Potential targets for colorectal cancer prevention. Int J Mol Sci. 2013;14:17279-303.

28. Strillacci A, Griffoni C, Sansone P, et al. MiR-101 downregulation is involved in cyclooxygenase- 2 overexpression in human colon cancer cells. Exp Cell Res. 2009;315:1439-47.

29. Roelofs HM, Te Morsche RH, an Heumen BW, Nagengast FM, Peters WH. Over-expression of COX-2 mRNA in colorectal cancer. BMC Gastroenterol. 2014;14:1.

30. Patrignani P, Patrono C. Aspirin and Cancer. J Am Coll Cardiol. 2016;68:967-76.

31. Brown JR, DuBois RN. COX-2: a molecular target for colorectal cancer prevention. J Clin Oncol. 2005;23:2840-55.

32. Cao Y, Nishihara R, Qian ZR, Song M, et al. Regular aspirin use associates with lower risk of colorectal cancers with low numbers of tumor-infiltrating lymphocytes. Gastroenterology. 2016; 151:879-92.e4.

33. Zelenay S, an der Veen AG, Bottcher JP, et al. Cyclooxygenasedependent tumor growth through evasion of immunity. Cell. 2015;162:1257-70.

34. Rothwell PM. Aspirin in prevention of sporadic colorectal cancer: current clinical evidence and overall balance of risks and benefits. Recent Results Cancer Res. 2013;191:121-42.

35. Veettil SK, Lim KG, Ching SM, Saokaew S, Phisalprapa P, Chaiyakunapruk N. Effects of aspirin and non-aspirin nonsteroidal anti-inflammatory drugs on the incidence of recurrent colorectal adenomas: a systematic review with meta-analysis and trial sequential analysis of randomized clinical trials. BMC Cancer. 2017; 17:763.

36. Elzey BD, Tian J, Jensen RJ, et al. Platelet-mediated modulation of adaptive immunity. A communication link between innate and adaptive immune compartments. Immunity. 2003;19:9-19.

37. Sitia G, Aiolfi R, Di Lucia P, et al. Antiplatelet therapy prevents hepatocellular carcinoma and improes survival in a mouse model of chronic hepatitis B. Proc Natl Acad Sci USA. 2012;109: E2165-72.

38. Rachidi S, Metelli A, Riesenberg B, et al. Platelets subvert T cell immunity against cancer via GARP-TGFbeta axis. Sci Immunol, 2017;2:1-27.

39. Ruschoff J, Wallinger S, Dietmaier W, et al. Aspirin suppresses the mutator phenotype associated with hereditary nonpolyposis colorectal cancer by genetic selection. Proc Natl Acad Sci USA. 1998;95:11301-6.

40. McIlhatton MA, Tyler J, Burkholder S, et al. Nitric oxidedonating aspirin deriaties suppress microsatellite instability in mismatch repair-deficient and hereditary nonpolyposis colorectal cancer cells. Cancer Res. 2007;67:10966-75. 
41. Luciani MG, Campregher C, Gasche C. Aspirin blocks proliferation in colon cells by inducing a G1 arrest and apoptosis through actiation of the checkpoint kinase ATM. Carcinogenesis. 2007;28:2207-17.

42. Schmugge M, Speer O, Kroiss S, et al. Monitoring aspirin therapy in children after interventional cardiac catheterization: laboratory measures, dose response, and clinical outcomes. Eur J Pediatr, 2015;174:933-41.

43. Schoenfeld AJ, Grady D. Adverse effects associated with proton pump inhibitors. JAMA Intern Med. 2016;176:172-4.

44. Lazarus B, Chen Y, Wilson FP, et al. Proton pump inhibitor use and the risk of chronic kidney disease. JAMA Intern Med. 2016;176:238-46.

45. Howell MD, Noack V, Grgurich P, et al. Iatrogenic gastric acid suppression and the risk of nosocomial Clostridium difficile infection. Arch Intern Med. 2010;170:784-90.

46. Belay ED, Bresee JS, Holman RC, Khan AS, Shahriari A, Schonberger LB. Reye's syndrome in the United States from 1981 through 1997. N Engl J Med. 1999;340: 1377-82.

47. Autret-Leca E, Jonille-Bera AP, Llau ME, et al. Incidence of Reye's syndrome in France: a hospital-based surey. J Clin Epidemiol. 2001;54:857-62.

48. Hurwitz ES, Barrett MJ, Bregman D, et al. Public Health Serice study of Reye's syndrome and medications. Report of the main study. JAMA. 1987;257:1905-11.
49. Barrett MJ, Hurwitz ES, Schonberger LB, Rogers MF. Changing epidemiology of Reye syndrome in the United States. Pediatrics. 1986;77:598-602.

50. Hurwitz ES, Barrett MJ, Bregman D, et al. Public Health Service study on Reye's syndrome and medications. Report of the pilot phase. N Engl J Med. 1985;313:849-57.

51. Smith ET Jr., Dais GJ. Medium-chain acylcoenzyme-A dehydrogenase deficiency. Not just another Reye syndrome. Am J Forensic Med Pathol. 1993;14:313-8.

52. Gosalakkal JA, Kamoji V. Reye syndrome and reye-like syndrome. Pediatr Neurol. 2008;39:198-200.

53. Schror K. Aspirin and Reye syndrome: a review of the evidence. Paediatr Drugs. 2007;9:195-204.

54. Gioannucci E. The prevention of colorectal cancer by aspirin use. Biomed Pharmacother. 1999;53:303-8.

55. Eleftheriou D, Lein M, Shingadia D, Tulloh R, Klein NJ, Brogan PA. Management of Kawasaki disease. Arch Dis Child. 2014;99:74-83.

56. Anderson BJ, Holford NH. Mechanism-based concepts of size and maturity in pharmacokinetics. Annu Re Pharmacol Toxicol. 2008;48:303-32.

57. Cascorbi I. Drug interactions-principles, examples and clinical consequences. Dtsch Arztebl Int. 2012;109:546-55. quiz 556

58. Nalamachu S, Pergolizzi JV, Raffa RB, Lakkireddy DR, Taylor R Jr. Drug-drug interaction between NSAIDS and low-dose aspirin: a focus on cardioascular and GI toxicity. Expert Opin Drug Saf. 2014;13:903-17. 